\title{
An Open and Reconfigurable Wireless Sensor Network for Pervasive Health Monitoring
}

\author{
A. Triantafyllidis, V. Koutkias, I. Chouvarda and N. Maglaveras \\ Lab of Medical Informatics \\ Faculty of Medicine, Aristotle University of Thessaloniki \\ Thessaloniki, Greece \\ \{atriant, bikout, ioanna, nicmag\}@med.auth.gr
}

\begin{abstract}
Sensor networks constitute the backbone for the construction of personalized monitoring systems. Currently, a plethora of sensor networks for diverse pervasive healthcare applications has been proposed and implemented. However, there is a significant lack of open architectures in the field, resulting in closed, non-interoperable, and difficult to extend solutions. In this context, we propose an open and reconfigurable Wireless Sensor Network (WSN) for pervasive health monitoring, with particular emphasis in its easy extension with additional sensors and functionality by incorporating embedded intelligence mechanisms. We consider a generic WSN architecture comprised of diverse sensor nodes (with communication and processing capabilities) and a Mobile Base Unit (MBU) operating as the gateway between the sensors and the medical personnel, formulating this way a Body Area Network (BAN). The primary focus of this work is on the intra-BAN data communication issues, adopting SensorML as the data representation mean. Our prototype implementation illustrates the underlying design conceptualization and virtue of the proposed approach.
\end{abstract}

Keywords-Wireless sensor networks; Open architecture; SensorML; Pervasive healthcare; Embebbed intelligence

\section{INTRODUCTION}

Advances in Information and Communications Technologies have led to a number of available services and devices for pervasive health monitoring [1]. The primary goals of research and industry efforts are to facilitate clinicians in adopting the correct patient health treatment plan, to help patients continue with their daily life activities by making their monitoring and treatment services unobtrusive, and to provide appropriate alert mechanisms in case of emergencies [2]. Towards this direction, small and fast sensor platforms comprised of microcontroller-transceiver modules with sensing capabilities have been designed for ubiquitous health monitoring. Such autonomous distributed devices can be incorporated in Wireless Sensor Networks (WSNs) [3]. Current sensor applications, however, face the problem of being tightly coupled with the sensor hardware technology involved, resulting in proprietary solutions.

In order to incorporate such WSNs in healthcare service provision, quality and standardization issues have to be taken into account. In addition, performance and extensibility properties, as well as interoperability are crucial. Hence, an open architecture and standard ways of exchanging information and managing services in the application level are needed, in order to integrate both sensor platforms and Body Area

Permission to make digital or hard copies of all or part of this work for personal or classroom

use is granted without fee provided that copies are not made or distributed for profit or commercial

advantage and that copies bear this notice and the full citation on the first page. To copy otherwise,

to republish, to post on servers or to redistribute to lists, requires prior specific permission and/or a fee.

PERVASIVEHEALTH 2008, 30 Jan - 1 Feb. Tampere, Finland

Copyright $\odot 2008$ ICST 978-963-9799-15-8

DOI 10.4108/ICST.PERVASIVEHEALTH2008.2522
Networks (BANs) in intra-BAN communication and extraBAN communication, respectively. Adoption of open architectures can facilitate the integration of sensor node services in the existing medical services infrastructure. Several projects, such as MobiHealth [4], focus on Service Oriented Architectures (SOAs) and open standards. In these approaches, sensor nodes are not considered as able to embed intelligence, but rather as simple data sources that forward data towards a single sink, i.e., a PDA, maximizing in this way the communication cost.

In this context, a WSN which provides open and extensible mechanisms for a dynamic and reconfigurable network is presented. We are inspired from the Sensor Web Enablement (SWE) framework defined by the Open Geospatial Consortium (http://www.opengeospatial.org), which envisions Web resident sensors that are easily discoverable and accessible through the Internet, aiming to exploit sensor-based applications into Grid computing infrastructures. In particular, the focus of this work resides in the intra-BAN architecture and data communication, which is the base for the realization of such a generalized framework. In our approach, processing and communication capabilities are not residing in a single control unit, i.e., in a centralized manner. Instead, the WSN consists of sensor nodes able to apply appropriate data processing algorithms, in order to distinct normal and problematic situations in a patient's condition and send data only when needed. This processing burden is indispensable, since current sensor nodes with advanced processing capabilities can tolerate it, something that is untrue for the communication cost.

There are three basic qualities in the proposed architecture:

Formal sensor descriptions: Sensors provide standard selfdescriptions and processes, so as the entire network can be sufficiently described. For this reason, the SensorML [5] model language is adopted. SensorML, even if complex and rather heavyweight, can describe sensors and processes inside the network in a dynamic manner.

Openness: Sensors can be easily added or removed from the network, since the nature of sensor networks in healthcare can change over time for a particular patient. Adding a new sensor in a BAN is supported in a plug ' $n$ play mode, provided that the sensor is self-described via a valid SensorML document, preserving this way the unobtrusive operation of the network.

Reconfigurable network: Sensors can be reconfigurable in run-time, in the sense of adapting their operation logic. For 
example, the sampling frequency of a physiological parameter monitored may have to change, depending on certain personalized criteria, such as age and medical history, or even during emergencies. Personalized thresholds for parameters of a physiological phenomenon should also be reconfigurable. This requirement is also met by using SensorML as a means to define the sensors' operation logic.

The methodology followed to implement the abovementioned quality attributes as well as a prototype implementation scenario are presented in the following.

\section{MATERIALS AND METHODS}

\section{A. Overview}

In Fig. 1, the proposed system architecture is illustrated. The primary objective of this system is to provide a dynamic, adaptive and autonomous functionality in healthcare monitoring applications via an advanced WSN. Specifically, the BAN behavior is dynamically updated according to the requirements specified by the Healthcare Professionals. Moreover, the BAN is able to provide appropriate, formal selfdescriptions of the incorporated sensors, the processes conducted, and the overall network description, so as to ease later discovery and assure interoperability.

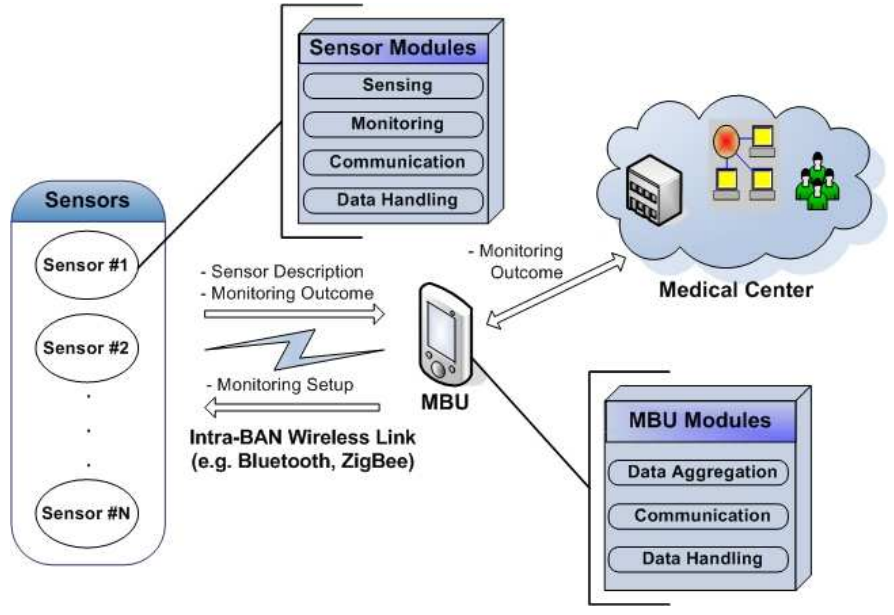

Figure 1. Overall system architecture.

We assume that the WSN is used as a personalized monitoring system providing its outcome to a Medical Center; however, in this work we particularly focus on the intra-BAN data communication aspects. Asynchronous ways of communication like SMS/MMS are currently investigated for providing appropriate notifications to the medical personnel, according to the monitoring outcome, aiming to close the loop between the patient and the healthcare provider. As for the data management issues in the Medical Center site, we have presented in [6] a multiagent system highlighting interesting patterns of patient interaction with a Medical Contact Center for chronic disease management, among which the deteriorization in patients' health according to the bioparameters monitored.

The entire intra-BAN data communication relies on XML standard mechanisms that are employed for representing data and formatting messages [3]. Although this is a heavyweight approach, XML provides efficient means for data description, query and task conduction due to its high degree of flexibility. Hence, SensorML is adopted so as to provide a generic data model for sensors description and functionality. The architecture relies on the Mobile Base Unit (MBU), which operates as the gateway between the sensor network and Healthcare Professionals. The MBU device has more advanced processing and networking capabilities than the sensors, making in this way possible to apply SensorML-based serialization and de-serialization of data, to coordinate network tasks, and communicate with sensors in an efficient manner.

\section{B. The Sensor Node}

The sensor node as shown in Fig. 1 is comprised of four main modules. The sensing module is responsible to receive physiological data associated with the sensor, while the monitoring module processes the sensing data according to the specified parameters. The communication module provides effective wireless communication with the MBU and, finally, the data handling module supplies efficient mechanisms for serialization and de-serialization of data. The sensor description is an important feature towards building a BAN with dynamic nature. This is achieved through a SensorML-based selfdescription of the sensor, which is sent to the MBU the first time it connects to it, embedding all the information that the MBU needs to be aware of. Specifically, the description contains identification information, information for the detector(s) that the node is consisted of, the type of physiological signal measured, etc. The description of a heart rate sensor node is depicted in Fig. 2, highlighting e.g., the resolution and accuracy capabilities of the sensor, the heartRate input and averageAlert output, the minAlertRuleTrigger process supported, etc.

\section{C. $M B U-$ Sensor Communication}

Wireless connectivity is provided for the communication between the MBU and the sensor nodes, since the use of wires cannot be considered as a user-friendly approach for an active patient/citizen. The MBU consists of the data aggregation module, the communication module and the data handling module. The MBU captures the description of each connected sensor and creates an adaptive-to-description user interface (UI) which helps Healthcare Professionals configure the monitoring parameters according to the patient's profile. The UI encapsulates information regarding sensors capabilities, so as to provide the potential of structuring appropriate medical rules for the sensors with personalized criteria, as described in the next section. After setting-up a medical rule, the rule is encoded in SensorML and sent to the sensors involved. Thus, the functionality of the sensors is adapted, with respect to the monitoring procedure. This means that the monitoring module of each sensor is dedicated to the specified rule and is responsible for applying the corresponding algorithm.

\section{WSN Intelligence}

In our design, where processing capabilities are assumed for both sensors and the MBU, intelligence is distributed in all nodes of the WSN, so as to operate in an overall efficient 
manner, enabling the nodes to process incoming information and act accordingly. Thus, events and actions triggered by events are described for each node of the WSN leading to different severity levels. Currently, we have elaborated on spontaneous events, i.e., monitoring whether a raw signal value or signal derivative, average value, maximum or minimum value etc, is above or below predefined thresholds within a time window, while a more challenging goal is to monitor and handle persistent ones characterized by the event onset and event end. In both cases of events, actions are triggered in the form of messages to the MBU, describing the event and optionally carrying the data that triggered the event. The sensors transmit information to the MBU only according to such customizable rules described in the form of event-action. An example rule involving heart rate or blood glucose average values within a specific time window is presented in Fig. 3.

The MBU receives information from multiple sensors and, since it has higher processing capabilities, it incorporates more sophisticated logic. Compound rules that combine information about multiple biosignals are supported in the MBU. The actions in this case can be either respond to the sensor, generate an alert for the system user, or process and forward information to the Medical Center. Beyond typical IF-THEN rules currently applied, more sophisticated data fusion methods are considered. Furthermore, a second level of prioritization can be introduced, according to the significance of multiple alerts [7].

\section{PROTOTYPE IMPLEMENTATION}

In our prototype implementation two sensor nodes are emulated; one for Heart Rate monitoring and the other for Blood Glucose observations. The sensor nodes are assumed to be resource-constrained mobile terminals. Java 2 Micro Edition (J2ME) with Connected Limited Device Configuration (CLDC) [8] is used to implement both the sensor nodes and the MBU components. J2ME with CLDC is an open platform for the development of mobile services and is currently supported by Sun SPOT sensor platform (http://www.sunspotworld.com) and the majority of mobile devices (e.g., PDAs, mobile phones, etc). CLDC configuration defines a virtual machine for resource-constrained devices together with a basic set of functionalities, providing in this way some of the flexibility of a complete computing system.

Bluetooth is chosen as the short-range wireless communication link for the intra-BAN communications, as a low-cost and low-power radio interface, used widely in a number of mobile devices and sensor nodes. JSR-82 libraries (http://jcp.org/en/jsr/detail?id=82) are used as the necessary interface that link Bluetooth hardware to Java and support important functionality, like ad-hoc device connection and automatic service discovery. Serialization and de-serialization of data is achieved using the kXML package (http://kobjects.org/kxml/), providing a lightweight, nonvalidating pull-parser that is ideal for the targeted mobile terminals. Therefore, SensorML messages can be generated and parsed efficiently.

The Bluetooth Service Discovery Protocol (SDP) provides a way of discovering services on a particular device. A Bluetooth-enabled device can act as a server and/or client. For this prototype implementation, three Bluetooth services are used. Firstly, a Send_Description service provided by the sensor nodes, which is used for sending the appropriate sensor self descriptions to the MBU. Secondly, a Send_Medical_Rules service provided by the MBU is used for sending medical rules to sensor nodes. Finally, the Send_Alert service is used for sending Bluetooth alerts to the MBU, when the criteria of medical rules are met. As far as security issues are concerned, we currently employ the built-in authentication mechanism of Bluetooth, while we consider data encryption techniques on top of the Bluetooth protocol stack as a more advanced security approach.

$<$ Sensor id="HR_id" $>$

$<$ identification $><$ identifierList $><$ identifier name="shortName"/><identifier name="longName"/><identifier name="modelNumber"/><identifier name="manufacturer"/ $><$ /identifierList $><$ /identification $>$

$<$ !-- Sensor general and functional capabilities -->

$<$ capabilities $><$ PropertyList $><$ property name="generalCapabilities" $><$ DataGroup $><$ component name $=$ "resolution" $>1.0</$ component $>$

$<$ component name="range" $>0200</$ component $><$ component name="accuracy" $>-11</$ component $></$ DataGroup $><$ /property $>$

$<$ !-- Sensor functional capabilities for samplingRate and sizeOfWindow configuration -->

$<$ property name="functionalCapabilities" $><$ DataGroup $><$ component name="samplingRateRangeConfigurationCapability" $>$

$0.0011<$ component $><$ component name="sizeOfWindowRangeConfigurationCapability" $>$

$1100</$ component $></$ DataGroup $><$ /property $><$ /PropertyList $></$ capabilities $>$

$<$ !--Sensor inputs-->

$<$ inputs $><$ inputList $><$ input name="heartRate" $><$ input $><$ inputList $><$ /inputs $>$

$<$ !--Sensor outputs-->

$<$ outputs $><$ outputList $><$ output name="minAlert" $><$ DataGroup $><$ component name="eventTime" $></$ component $><$ component name $=$ "measuredValue" $>$ $</$ component $></$ DataGroup $></$ output $><$ output name="maxAlert" .../><output name="averageAlert" .../ $></$ outputList $></$ outputs $>$

$<$ !--Definition of the actuating procedure of an alert with MIN criteria, as a process model-->

$<$ processes $><$ processList $><$ process name $=$ "minAlertRuleTrigger" $>$

$<$ inputs $><$ inputList $><$ input name="heartRate" $><$ /input $><$ inputList $><$ /inputs $>$

$<$ outputs $><$ outputList $><$ output name $=$ "eventTime" $><$ output $><$ output name="measuredValue" $>$

$<$ /output $><$ /outputList $><$ /outputs $><$ parameters $><$ ParameterList $><$ parameter name="heartRateMin" $><$ Datagroup $>$

$<$ component name="comparisonCriteria"/ $><$ component name="maxThreshold"/ $>$

$<$ component name="timeFrame"/ $><$ /Datagroup $><$ /parameter $><$ ParameterList $>$

$<$ parameters $><$ /process $>\ldots<$ /processList $><$ /processes $>$

$<$ !-- System inputs to process inputs, process outputs to system outputs -->

$<$ connections $><$ ConnectionList $><$ connection name="outputOfMinAlertRuleTriggerToSensor" $><$ Link $>$

$<$ source ref="minAlertRuleTrigger/outputs/measuredValue"/ $><$ destination ref="this/outputs/minAlert/measuredValue"/ $></$ Link $>$

$</$ connection $>$... $</$ ConnectionList $></$ connections $></$ Sensor $>$

Figure 2. Example SensorML-based description of a heart rate sensor. 


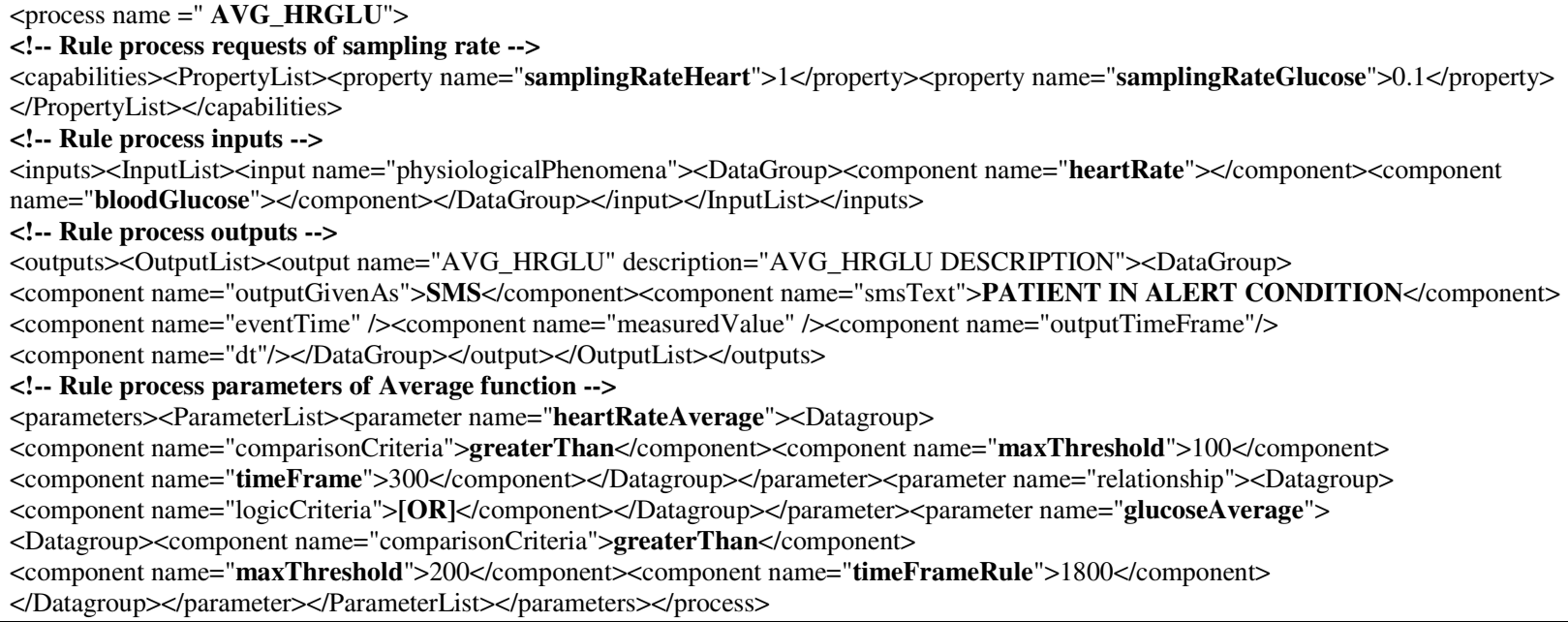

Figure 3. Example SensorML-based rule defining the generation of an SMS-action in case the average heart rate or blood glucose value exceeds a threshold within a specific time window.

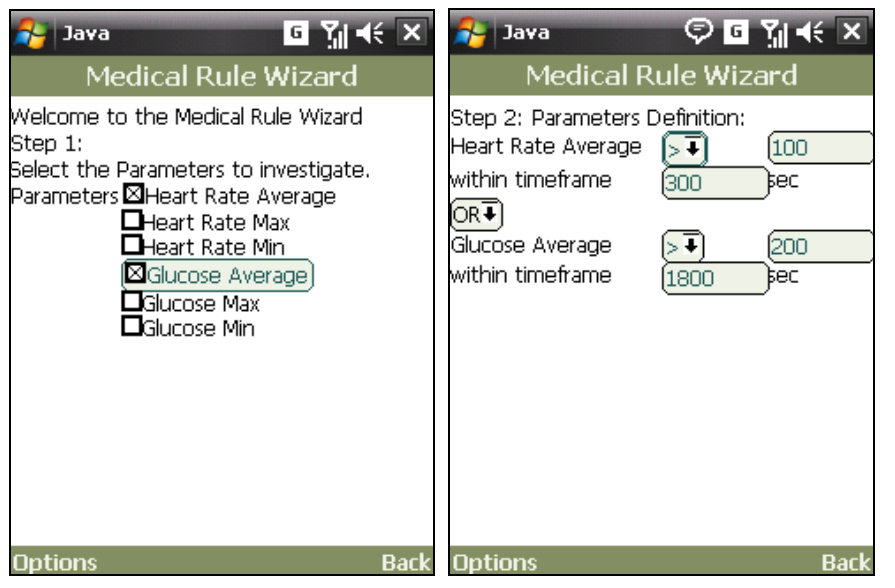

Figure 4. Adaptive user interface for medical rules definition through a stepby-step procedure.

The MBU (in our case, a Qtek ${ }^{\mathrm{TM}}$ MDA II PDA) parses the SensorML descriptions received via the Send_Description service and finds the potential functional capabilities of the Heart Rate and Blood Glucose sensors. An adaptive UI is then built based on these capabilities as shown in Fig. 4. The sensors are assumed to have MIN, MAX and AVERAGE calculation capabilities for a specified time frame. Health Professionals can then specify the parameters that these capabilities imply, via a step-by-step wizard, through which the rules of interest are determined. Note that the right-hand image corresponds to the SensorML-based rule depicted in Fig. 3.

\section{DISCUSSION}

Wireless sensor networks constitute a significant technological advance towards the construction of personalized health monitoring systems. The majority of WSN systems that are currently available targeting pervasive healthcare scenarios are rather application-specific, closed, non-interoperable, and difficult to extend. In this context, we propose an open and reconfigurable WSN for pervasive health monitoring, with particular emphasis in its easy extension with additional sensors and functionality by supporting embedded intelligence mechanisms. We consider a generic WSN architecture comprised of diverse sensor nodes and a gateway between the sensors and the medical personnel (MBU), formulating this way a Body Area Network (BAN). The primary focus of this work is on the intra-BAN data communication issues, adopting SensorML as the data representation mean in all transactions.

Our future development involves the formalization of the monitoring patterns as the embedded intelligence mechanism in the sensors, aiming to support both spontaneous and persistent events identification and management, as well as the relevant data aggregation issues that arise. Our ultimate goal is the implementation of closed-loop monitoring schemas by interconnecting the WSN with the Medical Center environment. The work presented constitutes an essential step towards the construction of open, extensible, interoperable, and intelligent WSNs for pervasive healthcare service provision.

\section{REFERENCES}

[1] U. Varshney, "Pervasive healthcare," IEEE Computer, vol. 36, no. 12, Dec. 2003, pp. 138-140.

[2] G. Borriello, V. Stanford, C. Narayanaswami, and W. Menning, "Pervasive computing in healthcare," IEEE Pervasive Computing, vol. 6, no. 1, Jan.-Mar. 2007, pp. 17-19.

[3] M. Balazinska et al., "Data management in the worldwide sensor Web," IEEE Pervasive Computing, vol. 6, no. 2, April-June 2007, pp. 30-40.

[4] K. E. Wac et al., "Mobile health care over 3G networks: The MobiHealth pilot system and service," Proc. of Global Mobile Congress, Shanghai, 2004, pp. 71-76.

[5] OGC ${ }^{\circledR}$ SensorML, http://www.opengeospatial.org/standards/sensorml/

[6] V. G. Koutkias, I. Chouvarda, and N. Maglaveras, "A multiagent system enhancing home-care health services for chronic disease management," IEEE Trans Inf Technol Biomed, vol. 9, no. 4, pp. 528-537, 2005.

[7] R .- G. Lee, K. -C. Chen, C. - C. Hsiao, and C.- L. Tseng, "A mobile care system with alert mechanism," IEEE Trans Inf Technol Biomed, vol. 11, no. 5, pp. 507-517, 2007.

[8] Java ME: Connected Limited Device Configuration (CLDC); JSR 30, JSR 139, Sun Microsystems, http://java.sun.com/products/cldc/ 